Check for updates

1 Royal College of Psychiatrists

2 South London and Maudsley NHS Foundation Trust

Cite this as: BMJ 2022;376:012 http://dx.doi.org/10.1136/bmj.o12 Published: 5 January 2022

\title{
If the government is serious about tackling inequalities, the Health and Care Bill must get serious about data
}

\author{
Lade Smith, ${ }^{1,2}$ Rajesh Mohan ${ }^{1,2}$
}

The National Health Service was founded to improve the nation's health and wellbeing. We know that all too often it has failed to do so equally for all the different communities it serves. Those at increased risk of developing a mental illness because of the disadvantages they face in everyday life are also less likely to access services and more likely to face poor outcomes when they do. The financial implications of our failure to tackle health inequalities are staggering and we simply cannot afford to continue turning a blind eye.

Simon Wessely's review of the Mental Health Act heard that people from Black African and Caribbean heritage have longer hospital stays, higher rates of repeat admissions and seclusion, and are less likely to be offered psychological therapies. Many LGBTQ+people avoid healthcare settings because of the discrimination they face, despite being at increased risk of common mental illnesses. People in lower income households are more likely to have unmet mental health treatment requests compared to those with the highest incomes. This is unjust and unacceptable.

Current NHS legal duties on tackling inequalities were brought into force with the National Health Service Act 2006 and the Equality Act 2010. Our collective awareness around inequalities has grown considerably in recent years and progress has no doubt been made, but it has been patchy and insufficient. In fact, the small volume and poor quality of inequalities data in mental health means that assessing where and how much progress has been made is challenging.

The Royal College of Psychiatrists' Equality Action Plan includes a long list of commitments to tackle inequalities through our work with members, health services, and government, but the lack of quality data will continue to hamper progress. ${ }^{1}$ Improving the volume and quality of data on protected characteristics across the Mental Health Services Data Set would dramatically increase our understanding of mental health inequalities and how we might tackle them.

NHS England/Improvement's 2020 Advancing Mental Health Equalities Strategy dedicates an entire workstream to improving the availability and quality of data and information. ${ }^{2}$ As health system leaders have struggled to move the needle up until now, the strategy could support progress at a local and regional level. Further guidance on inequalities data and central oversight of its implementation across local areas would certainly drive change. But with the new Health and Care Bill on the agenda in Parliament, we have a unique opportunity for a new legal framework that ensures NHS tackles inequalities head on.

The proposed legislation already includes duties for Integrated Care Boards to "have regard" to developing services that address inequalities in both access and outcomes. However, this is relatively similar to the duties currently placed on Clinical Commissioning Groups, and the Bill must go further to recognise how central tackling inequalities is to delivering on the NHS' core mission of improving health and wellbeing for all. An amendment to the new legislation requiring local systems to identify and monitor inequalities in health in their local population could help drive this issue to the core of local NHS planning. Strengthened emphasis on implementing stronger systems to understand local health inequalities would ensure that Integrated Care Systems (ICSs)s are able to target and track interventions.

Integrated commissioning and closer collaboration between the NHS, local councils, and social care providers also opens up an opportunity to build accessible and appropriate mental health services for those most disadvantaged and discriminated against. The increased focus on inequalities by authorities like the Care Quality Commission will help strengthen accountability. Ultimately, however, their success in doing so will depend on services using this data to design and target meaningful interventions and measuring their effectiveness by analysing how the data change over time.

The proposed legal obligations for ICSs to proactively address inequalities must be strengthened to drive local areas to collect and report high-quality disaggregated data on health disparities. Without this, the risk of insufficient and ineffective action is still too great. This will not only cost those most disadvantaged by inequities, but also have an effect on the NHS' bottom line at a time when budgets are under unprecedented strain. It is high time we take health inequalities seriously, and better data must be the foundation of that effort.

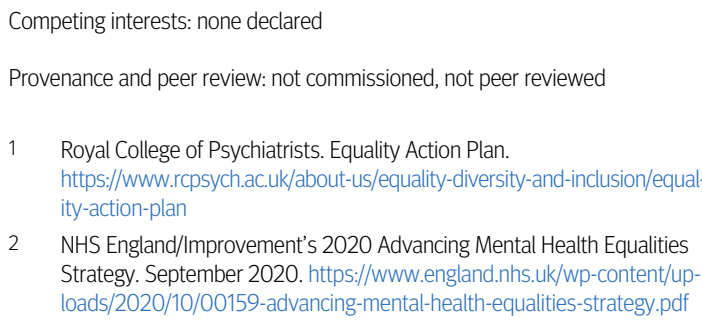

